Supplementary material for paper van der Heijden, Whittaker, Cruyff, Bakker and van der Vliet.

\title{
Estimation in $\mathrm{R}$
}

This document provides an example of the estimation procedure and the corresponding R-code to fit a log-linear model and obtain a confidence interval for the estimate of the population size $\mathrm{N}$. The example involves the log-linear model [AX2][BX1] to the data presented in Table 5 in the paper. In this example where $A$ and $B$ are the two incomplete registers, with covariate $X 1$ measured in $A$ but not in $B$ and covariate $X 2$ measured in B but not in A. The data are supposed to be in an SPSS file called "data.sav", with the missing values on the covariates coded as system missing.

The estimation procedure:

Step 1: reading the data into $R$

Step 2: fitting the log-linear model to the data with the missing covariates

Step 3: obtain data with imputation of the missing values of the covariates

Step 4: fitting the log-linear to the imputed data to obtain an estimate of $\mathrm{N}$

Step 5: calculation of the fit statistics

Step 6: parametric bootstrap to obtain the confidence interval for the estimate of $\mathrm{N}$

R-code:

\# loading the necessary libraries

library(cat)

library(foreign)

\# step 1: reading the data into $\mathrm{R}$

$\begin{array}{ll}\text { datafile } & <- \text { read.spss("data.sav", use.value.labels=FALSE,to.data.frame=TRUE) } \\ \text { colnames(datafile) } & <-c(" A ", " B ", " X 1 ", " X 2 ")\end{array}$

a $<-1$

b $<-2$

$\mathrm{x} 1<-3$

$x 2<-4$

\# specification of the log-linear model [AX2][BX1],

MODEL <- Freq $A^{*} \times 2+B * X 1$

MARGINS <- $(a, x 2,0, b, x 1)$

MAXMODEL <- Freq $\sim A^{*} X 2+B * X 1+X 1 * X 2$

\# step 2: fitting the log-linear model to the data with the missing covariates

table.miss <- as.data.frame(table(datafile))

observed <- ifelse(table.miss[,1] $==2$ \& table.miss[,2] $==2,0,1$ )

table.miss <- cbind(table.miss,observed)

dcat <- prelim.cat(as.matrix(datafile)) 
struc.zero <- array(observed, $\operatorname{dim}=$ dcat $\$ \mathrm{~d}) /$ sum(observed)

dcat.saturated <- em.cat(dcat,start=struc.zero,showits=FALSE)

dcat.model <- ecm.cat(dcat,start=struc.zero, margins=MARGINS, showits=FALSE,eps=1e-7)

\# Step 3: obtain data with imputation of the missing values covariates

$\begin{array}{ll}\text { table.complete } & <- \text { table.miss } \\ \text { table.complete\$Freq } & <- \text { as.numeric(dcat.mode }{ }^{*} \text { dcat } \$ n \text { ) }\end{array}$

\# Step 4: fitting the log-linear to the imputed data to obtain an estimate of $\mathrm{N}$

glm.complete $\quad<-$ gIm(MODEL,family=poisson, subset=(observed ==1), data = table.complete)

model.frame.complete <- model.frame(MODEL, data = table.complete)

fitted.complete <- predict(gIm.complete, model.frame.complete, type = "response")

hatN <- sum(fitted.complete)

\# Step 5: calculation of the fit statistics

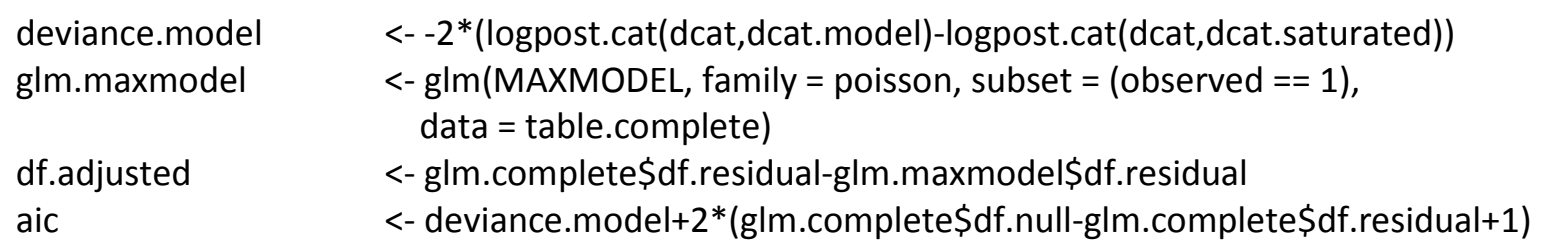

\# printing the results

results <- cbind(deviance.model,df.adjusted,aic,hatN)

colnames(results) <-c("Deviance","df","AIC","Pop.size")

rownames(results) <- c(MODEL)

print(results)

\# Step 6: parametric bootstrap to obtain the confidence interval for the estimate of $\mathrm{N}$

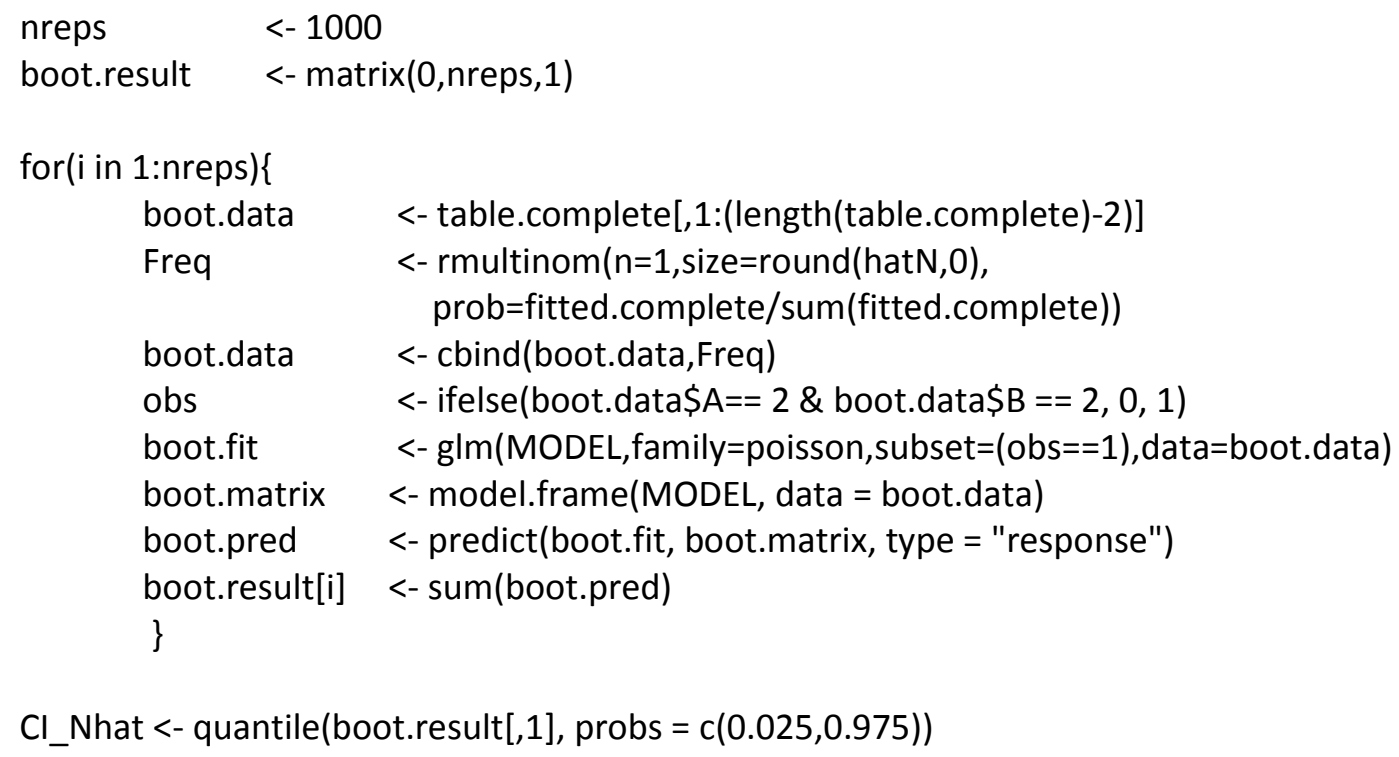

